\title{
Specific interaction of lectins with liposomes and monolayers bearing neoglycolipids
}

\author{
Vincent Faivre ${ }^{\mathrm{a}, 1}$, Maria de Lourdes Costa ${ }^{\mathrm{b}}$, Paul Boullanger ${ }^{\mathrm{c}}$, \\ Adam Baszkin $^{\text {a }}$, Véronique Rosilio ${ }^{\mathrm{a}, *}$ \\ a Physico-Chimie des Surfaces, UMR CNRS 8612, Université Paris-Sud, 5 rue J.B. Clément, \\ 92296 Châtenay-Malabry Cedex, France \\ ${ }^{\mathrm{b}}$ Farmacia Galenica, Faculdade de Farmacia, Universidade de Coimbra, Rua Norte, 3000 Coimbra, Portugal \\ ${ }^{c}$ UMR CNRS 5622, Université Lyon-I, CPE-Lyon, 43 boulevard du 11 Novembre 1918, 69622 Villeurbanne Cedex, France
}

Received 28 November 2002; received in revised form 26 May 2003; accepted 27 May 2003

\begin{abstract}
The interaction of three lectins (wheat germ, Ulex europaeus I, and Lotus tetragonolobus agglutinins: WGA, UEA-I and LTA) with either $N$-acetyl-D-glucosamine or L-fucose neoglycolipids incorporated into phospholipid monolayers and liposome bilayers was studied at the air/water interface and in bulk solution.

The results show that for both systems studied, synthesized neoglycolipids were capable of binding their specific lectin and that, in general, the binding of lectins increased with the increase in the molar fraction of the saccharide derivative incorporated in either the monolayers or bilayers. However, whereas for UEA-I, molecular recognition was enhanced by a strong hydrophobic interaction, for WGA and LTA successful recognition was predominantly related to the distance between neighboring sugar groups. The observed lengthy adsorption times of these lectins onto their specific ligands were attributed to interfacial conformational changes occurring in the proteins upon their adsorption at the interfaces.
\end{abstract}

(C) 2003 Elsevier Ireland Ltd. All rights reserved.

Keywords: Lectin; Glycolipid; Liposome; Monolayer; Surface pressure; Molecular recognition

\footnotetext{
Abbreviations: UEA-I, Ulex europaeus I agglutinin; WGA, wheat germ agglutinin; LTA, Lotus tetragonolobus agglutinin; DMPC, dimyristoylphosphatidylcholine

* Corresponding author. Tel.: +33-1-4683-5645; fax: $+33-1-4683-5312$.

E-mail address: veronique.rosilio@cep.u-psud.fr (V. Rosilio).

${ }^{1}$ Present address: Laboratoire de Recherche et Développement de Pharmacie Galénique Industrielle-ISPB, Université Lyon-I, 8 avenue Rockefeller, 69373 Lyon Cedex 08, France.
}

\section{Introduction}

A living cell is delimited by a membrane, which is the center of various functions (Sharon and Lis, 1993; Feizi, 1985; Kitano et al., 1998). Among them, many biological communications and recognition processes are mediated by interactions involving cell-surface glycolipids or glycoproteins (Roseman, 1970; Ashwell and Morell, 1974). The recognition of the sugar moieties by their specific proteins can induce a change in the three-dimensional structure of the interacting molecules. The structure of the carbohydrate moiety 
of molecules which are recognized by membrane lectins depends both upon the nature of cells and upon their affinity in key-lock specificities (Monsigny et al., 1979).

Although lectins were originally isolated from plants, proteins with lectin-like domains have also been identified in mammals, including humans (Kishore et al., 1997). They are involved in various functions such as glycoprotein endocytosis or pathogen lysis. Increasing evidence suggests that conformational properties of carbohydrates play an important role in the recognition phenomena that control the specificity of these processes (Hare et al., 1994). For example, the interaction between wheat germ agglutinin (WGA) and a sugar residue is dependent on the number of ethoxy units separating the headgroup of the sugar receptor from the alkyl chain. Ethoxy units added to receptor structure allow a headgroup to extend into the solution and they facilitate sugar interaction with specific lectins.

Lectin-sugar recognition systems have focused attention in the pharmaceutical field especially on the development of drug carriers tailored for selective delivery (Boldt et al., 1977; Boullanger et al., 1997; Chierici et al., 1997; Faivre et al., 2001; Irache et al., 1994; Ponchel and Irache, 1998). It seems possible to coat various drug delivery systems with either lectins or sugar moieties in order to improve their specificity and targetability. Sugar determinants on the surface of polymer nanoparticles or neoglycolipids incorporated into liposome bilayers hold promise for a more effective delivery of drugs (Boullanger et al., 1997; Chierici et al., 1997; Faivre et al., 2001). Also, one possible approach to improve oral bioavailability and efficiency of a drug is to resort to a bioadhesive system based on a receptor-mediated interaction within the gastrointestinal tract. In such an approach, lectins bound to polymer or protein nanoparticles recognize mucosaccharides (Irache et al., 1994; Ponchel and Irache, 1998). Until now, the use of liposomes in oral drug delivery has been limited because of their rapid dissolution by bile salts. Recently, Chen et al. (1996) overcame this difficulty using polymerized liposomes coated with lectins capable of targeting them to the Peyer's patch. Thus, a polysaccharide such as pullulan confers physical and biological stability to the liposomes. Sunamoto and co-workers demonstrated that the lectin-induced aggregation and the phagocyte uptakes of polysaccharide-coated liposomes might be effectively controlled by changing only the terminal sugar of the polysaccharide derivative (D-mannose, $\alpha$-D-glucose or $\beta$-galactose) (Akiyoshi et al., 1990). Also, Shimada et al. (1997) showed that liposome surface-exposed galactose residues, attached to the distal end of a poly(ethylene glycol) chain anchored in the liposomal bilayer were effectively recognized by the galactose particle receptor on the Kupffer cells. In another work, by Murahashi et al. (1997) it was reported that the recognition of neoglycolipid-bearing liposomes by liver cells took place. Apart from their ability to adsorb on a liposome surface and anchor to its bilayer membrane (Sancho et al., 1993), these neoglycolipids were found to be able to form supramolecular assemblies such as vesicles (Emmanouil et al., 1998; Blanzat et al., 1999), or nanoparticles formed of spherical sugar-persubstituted dendrimers (sugar balls) that exhibit a highly ordered structure with saccharide arrays on their periphery (Esumi et al., 2000). All these systems are not only potential drug carriers, but they provide also interesting artificial model systems for the studies of the mechanisms involved in molecular recognition. Also, Langmuir monolayers have successfully been used as simple models of lipidic cell membranes to mimic biological phenomena such as enzymatic action or molecular recognition (Berthelot et al., 1998; Kurihara et al., 1991; Ahlers et al., 1990; Baszkin and Norde, 1999).

The use of monolayers allows studying interactions at molecular level in the conditions where surface densities and packing of molecules are achieved in a controlled manner. They provide thus, complementary information to the experiments carried out in solution. In this paper, we report on the interfacial behavior of various neoglycolipids incorporated both in phospholipid monolayers and in liposome bilayers and on their interaction with specific lectins.

\section{Material and methods}

\subsection{Materials}

The neoglycolipids were formed of Guerbet alcohol $\left(\mathrm{G}_{28}\right)$ directly bound to a sugar moiety $(\mathrm{N}$-acetylglucosamine) or through a triethylene glycol $\left(\mathrm{E}_{3}\right)$ spacer $(N$-acetylglucosamine, $\alpha$ - or $\beta$-fucose). 


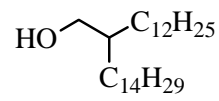

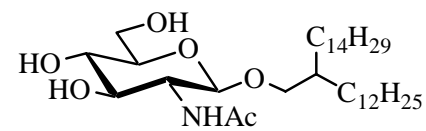<smiles>CCCCCCCCCCCCCCCCCCCCCOCCOCCOCCO</smiles>

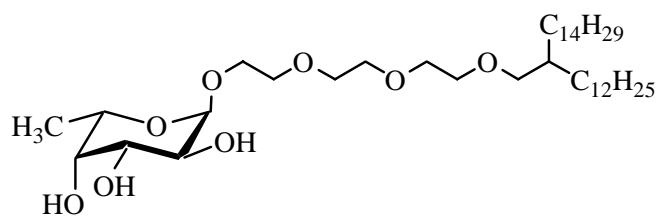

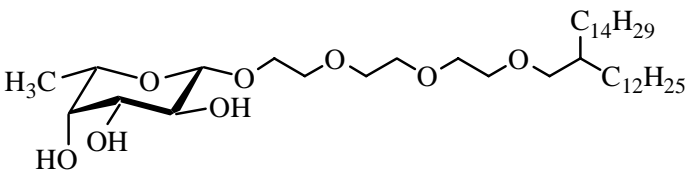

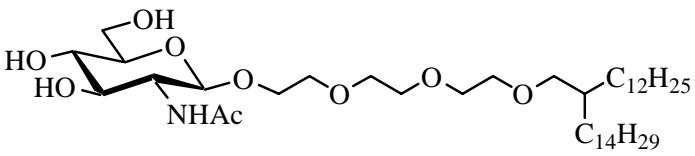

$\mathrm{G}_{28}$<smiles>[Mg][Mg][Mg]Cl</smiles>

$\mathrm{E}_{3} \mathrm{G}_{28}$

$\alpha-\mathrm{L}-\mathrm{FucE}_{3} \mathrm{G}_{28}$

$\beta-\mathrm{L}-\mathrm{FucE}_{3} \mathrm{G}_{28}$

$\mathrm{GlcNAcE}_{3} \mathrm{G}_{28}$

Fig. 1. Chemical structures of the studied lipids and neoglycolipids.

The $\beta$ - $N$-acetylglucosamine derivatives, GlcNAcG $_{28}$ $\left(M_{\mathrm{w}}\right.$ 613.97) and $\mathrm{GlcNAcE}_{3} \mathrm{G}_{28}\left(M_{\mathrm{w}}\right.$ 746.13), were synthesized according to the previously described route (Murahashi et al., 1997). The synthesis routes to fucose derivatives $\left(M_{\mathrm{w}} 689.08\right)$ led either to the pure $\beta$-FucE $\mathrm{E}_{3} \mathrm{G}_{28}$ or to a mixture of the $\alpha$ - and $\beta$-anomers (8.4:1.6), later referred to as $\alpha-\mathrm{FucE}_{3} \mathrm{G}_{28}$ in this paper (Faivre et al., 2001). All studied neoglycolipids (Fig. 1) were insoluble in water and soluble in the mixture of chloroform and methanol (9:1) used for spreading. Dimyristoylphosphatidylcholine (DMPC, $M_{\mathrm{w}}$ 677.94), and the lectins: WGA ( $M_{\mathrm{w}} 43,000$; Nagata and Burger, 1974), LTA (a mixture of the isolectins A $\left(M_{\mathrm{W}} 120,000\right), \mathrm{B}\left(M_{\mathrm{W}} 58,000\right)$ and $\mathrm{C}$ $\left.\left(M_{\mathrm{w}} 117,000\right)\right)$ and the UEA-I $\left(M_{\mathrm{w}} 68,000\right)$ were purchased from Sigma.

Milli-Ro 6+ ultrapure water was doubly distilled on acid $\mathrm{KMnO}_{4}$ before its use in all experiments. This wa- ter had a surface tension of $71.8 \pm 0.1 \mathrm{mN} / \mathrm{m}$ at $25^{\circ} \mathrm{C}$, in agreement with literature data. A $1 \mathrm{mM}$ HEPES solution, with or without $10^{-3} \mathrm{M}$ of $\mathrm{Ca}^{2+}, \mathrm{Mn}^{2+}$ and $\mathrm{Zn}^{2+}$ ions (pH 7.4) was used as a buffer. The glassware was cleaned in a freshly prepared sulfochromic solution and abundantly rinsed with ultrapure water.

\subsection{Liposome preparation and interaction with the lectins}

Liposome suspensions were prepared as previously described (Faivre et al., 2001) by extrusion of multilamellar liposome suspensions containing DMPC or DMPC/neoglycolipid mixtures through a polycarbonate membrane $(200 \mathrm{~nm})$. The size of liposome was measured by photon correlation spectroscopy on a Coulter submicron particle analyzer N4MD (Coultronics, Margency, France). The extent of the 
interaction between neoglycolipid bearing liposomes and the specific lectins was deduced from the experiments in which a lectin was added to a liposome suspension and the agglutination of the liposomes was monitored by measuring turbidity changes of suspensions.

\subsection{Surface tension experiments}

Surface tension measurements at equilibrium (constant area) were performed by the Wilhelmy plate method using a Krüss K10ST tensiometer (Germany) as previously described (Faivre et al., 2000). The measurements were taken without detaching the plate from the interface, and the data were continuously plotted on a calibrated chart recorder. All reported surface tension or pressure values are mean values of at least three measurements. The accuracy of the measurements was estimated to be $0.2 \mathrm{mN} / \mathrm{m}$. The surface pressures of the pure components or their mixtures in monolayers were deduced from the $\pi=\gamma_{0}-\gamma$ relationship, in which $\gamma_{0}$ is the surface tension of pure water and $\gamma$ is the surface tension observed in the presence of a monolayer. The surface pressure change $(\Delta \pi)$ corresponds to the increase in the surface pressure resulting from lectin injection beneath a spread monolayer.

\subsection{Surface pressure measurements}

A homemade Langmuir-type all-teflon micro-trough was used to record compression isotherms of the studied neoglycolipids and of neoglycolipid/DMPC monolayers. In these experiments, a chloroform-methanol $(9: 1, \mathrm{v} / \mathrm{v})$ solution containing the lipids was deposited onto the water subphase $\left(27 \mathrm{~cm}^{3}, 36 \mathrm{~cm}^{2}\right)$ by means of a micropipette (Microman Gilson) (Berthelot et al., 1998). All experiments were run at $22^{\circ} \mathrm{C}$. Experimental conditions were controlled to ensure limited water evaporation. In lectin-sugar interaction experiments, a neoglycolipid monolayer was spread onto the aqueous subphase so that the surface pressure could reach an initial surface pressure value of $9 \mathrm{mN} / \mathrm{m}$ $\left(\pi_{0}\right)$. It was considered that at this $\pi_{0}$ the neighboring neoglycolipid molecules have still enough freedom to orient specifically to interact with adsorbing lectins. The lectin solution was injected into the subphase beneath the monolayer and the solution was gently stirred for $5 \mathrm{~min}$. The increase in surface pressure due to the occurrence of the sugar-lectin interaction was time-dependent and the maximum $\pi$ value was reached after several hours. Then a mixed lectin/neoglycolipid monolayer was compressed at the rate of $4.8 \mathrm{~cm}^{2} / \mathrm{min}\left(0.3 \AA^{2}\right.$ per molecule/s). All reported results are mean values of at least three measurements. Deviations from the mean were less than $\pm 0.5 \mathrm{mN} / \mathrm{m}$.

\section{Results}

\subsection{Interaction of the lectins with DMPC/neoglycolipid liposomes}

The presence of neoglycolipid molecules in the liposomal membranes did not affect the size of the liposomes and the diameter of vesicles remained in all cases comprised between 165 and $176 \mathrm{~nm}$. A similar result has been previously reported with liposomes bearing in their walls $N$-acetylglucosamine derivatives (Sancho et al., 1993).

As it may be noted from Figs. 2a, c and e for all studied lectin/neoglycolipid systems, liposome agglutination increased with the molar fraction of the neoglycolipid incorporated in the phospholipid bilayer.

This finding is in agreement with literature data (Liener et al., 1986; Allen et al., 1977), which relate erythrocyte agglutination to the structure of the sugar $(\alpha$-or $\beta$-fucose). From our experiments it is clear that the level of liposome agglutination was much higher when UEA-I interacted with the liposomes bearing the $\alpha$-fucosyled neoglycolipid rather than with the $\beta$-fucosyled derivative (Fig. 2b). This is consistent with the already reported high specificity of UEA-I for the $\alpha$-anomer (Allen et al., 1977). As it is shown in Fig. 2d, LTA displayed a rather low specificity for fucosyled neoglycolipids.

The agglutination of $\mathrm{N}$-acetylglucosamine-bearing liposomes by WGA appeared to be greatly improved by an increase in the neoglycolipid/phospholipid ratio (Fig. 2e).

The data obtained strongly suggest that the mechanisms of molecular recognition between lectins and sugars vary from one system to the other and largely depend on the specific character of the studied 


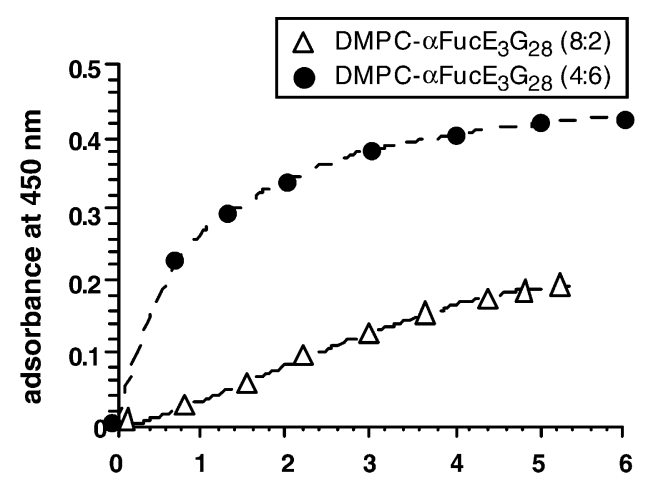

(a)

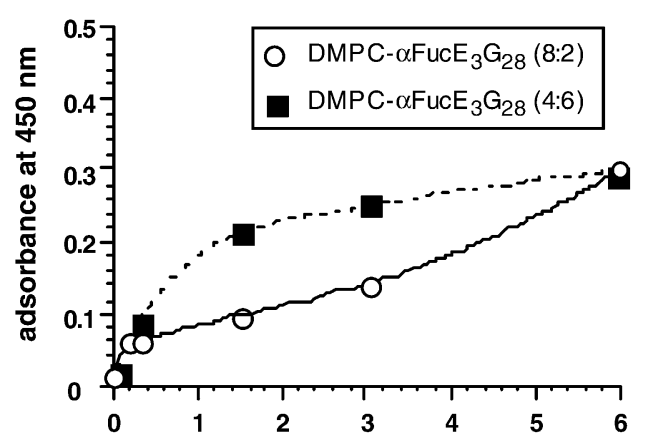

(c)

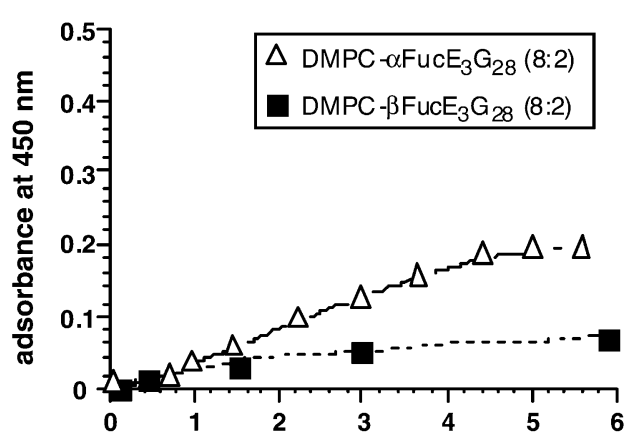

(b)

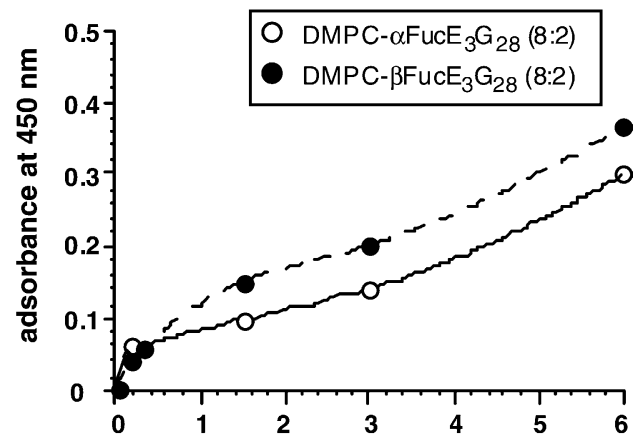

(d)

[LTA] $\mu \mathrm{M}$

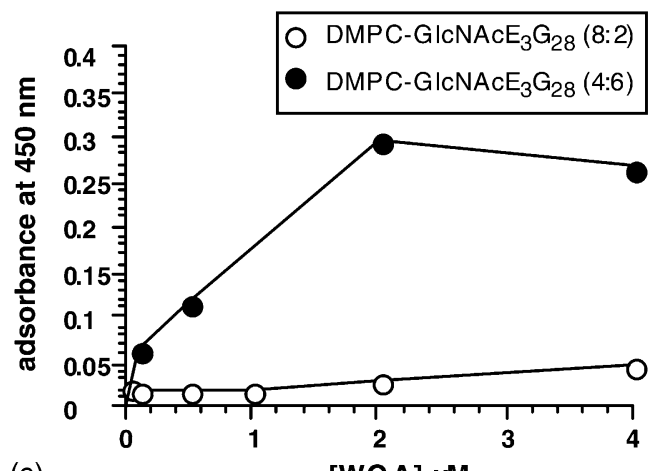

(e)

IWG A1 $1 \mathrm{M}$

Fig. 2. Changes in optical density of liposome suspensions induced by the presence of lectins.

molecules. In order to better understand these mechanisms, and to take into account the presence of non-specific interactions between the lectins and phospholipid molecules forming the liposome membrane, lectin-sugar interactions have been studied in a model system at the air/water interface.

\subsection{Interfacial behavior of the studied neoglycolipids}

The interfacial behavior of the neoglycolipids and of their mixtures with DMPC has already been described in our previous works (Faivre et al., 2001; Berthelot et al., 1998). Compared to the precursor lipid $\left(\mathrm{E}_{3} \mathrm{G}_{28}\right)$, 
the lipids with a sugar moiety attached to them display increasing molecular areas at pressures where the spread layers collapsed from their initial bidimensional structure parallel to the water plane interface to form tridimensional structures. These areas varied from $37.7 \AA^{2}$ (collapse pressure, $\pi_{\mathrm{c}}=39 \mathrm{mN} / \mathrm{m}$ ) for $E_{3} G_{28}$ to 52 and $55 \AA^{2}$ for the fucose derivatives $\left(\pi_{\mathrm{c}}=39.8\right.$ and $\left.28.5 \mathrm{mN} / \mathrm{m}\right)$, and up to $63 \AA^{2}\left(\pi_{\mathrm{c}}=\right.$ $34 \mathrm{mN} / \mathrm{m}$ ) for $\mathrm{GlcNAcE}_{3} \mathrm{G}_{28}$.

\subsection{Interfacial behavior of the lectins at the air/liquid interface}

The surface properties of the lectins were studied at different concentration ranges: for LTA at [1-10 nM], for UEA-I at [30-300 nM], and for WGA at $[0.1-3.5 \mu \mathrm{M}]$. The extent of their adsorption at the interface with air after a long period of time $(24 \mathrm{~h}$ for UEA-I and WGA, $40 \mathrm{~h}$ for LTA) was found to be highly dependent on the nature of the subphase buffer (Fig. 3). Whereas the presence of calcium, manganese and zinc cations in the buffer solution did not modify WGA adsorption, these cations lowered LTA adsorption and enhanced that of UEA-I.

The results of adsorption of the three lectins on DMPC monolayers, in the presence of divalent cations, are illustrated in Fig. 4. From this figure it is immediately apparent that the presence of a DMPC monolayer affects adsorption of the three studied lectins in a different manner. Whereas for WGA and LTA, a significant reduction in the extent of adsorption at high surface coverage was observed, for UEA-I the profile of $\Delta \pi$ versus $\pi_{0}$ exhibited a maximum.

\subsection{Adsorption of the lectins into DMPC/ \\ neoglycolipid monolayers at low initial surface pressure $\left(\pi_{0}=9 \mathrm{mN} / \mathrm{m}\right)$}

The extent of interaction between mixed monolayers and the lectins was inferred from the change in surface pressure $(\Delta \pi)$ following lectin injection beneath spread DMPC/neoglycolipid monolayers. At a given adsorption time $t, \Delta \pi$ represents the sum of the surface pressure due to both the specific interaction $\pi_{\mathrm{s}}$ (of the lectin with its ligand) and that due to the non-specific interaction $\pi_{\mathrm{ns}}$. Both non-specific and specific interaction contributions to lectin binding to the monolayers were studied independently.
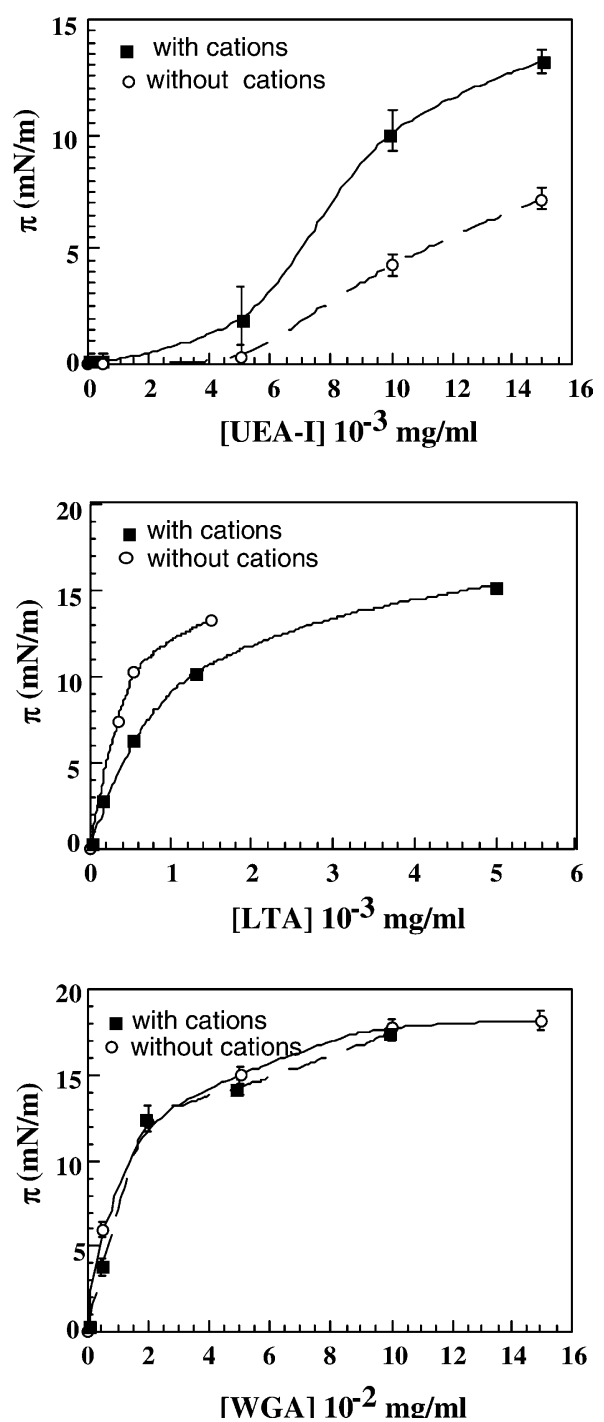

Fig. 3. Effect of the three divalent cations $\left(\mathrm{Zn}^{2+}, \mathrm{Mn}^{2+}\right.$ and $\left.\mathrm{Ca}^{2+}\right)$ on the interfacial behavior of the studied lectins.

The conditions of the sugar-lectin interaction were considered as non-specific when the lectin and sugar were non-specific to each other or in the absence of the divalent cations. Thus, for LTA and UEA-I non-specific sugars to the lectins were used whereas WGA/GlcNAcE ${ }_{3} \mathrm{G}_{28}$ interaction was studied in the absence of the divalent cations. The results of these experiments are presented in Fig. 5. They show that for each studied neoglycolipid/lectin system, the $\pi_{\mathrm{ns}}$ values are independent of the DMPC/neoglycolipid ratio. 


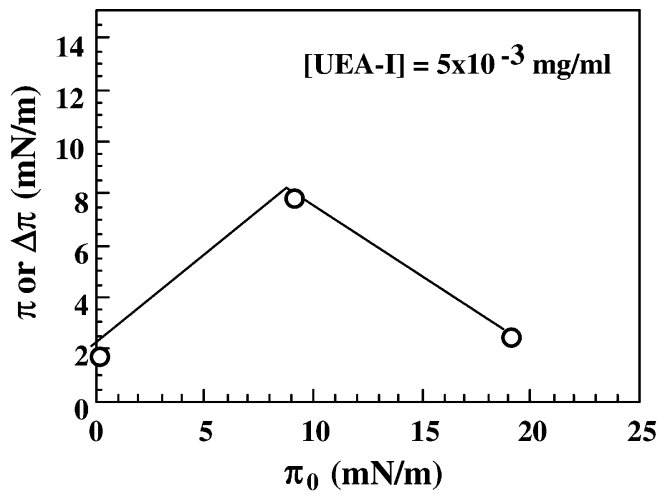

DMPC-GlcNAcE ${ }_{3} \mathrm{G}_{28}$ without divalent cations

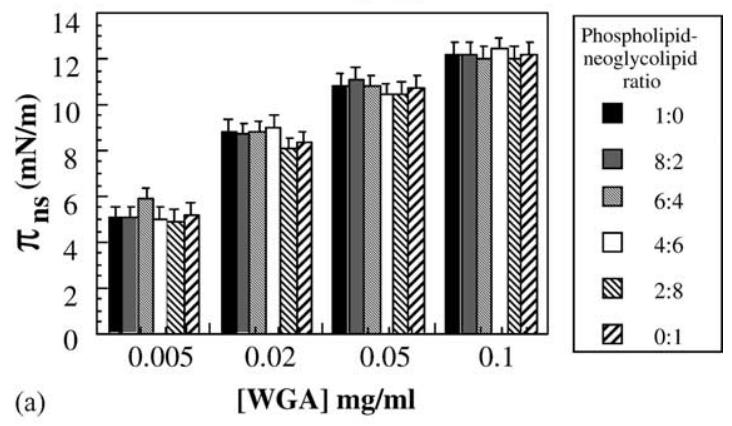

DMPC- $\beta$ FucE $_{3} \mathbf{G}_{28}$ with divalent cations
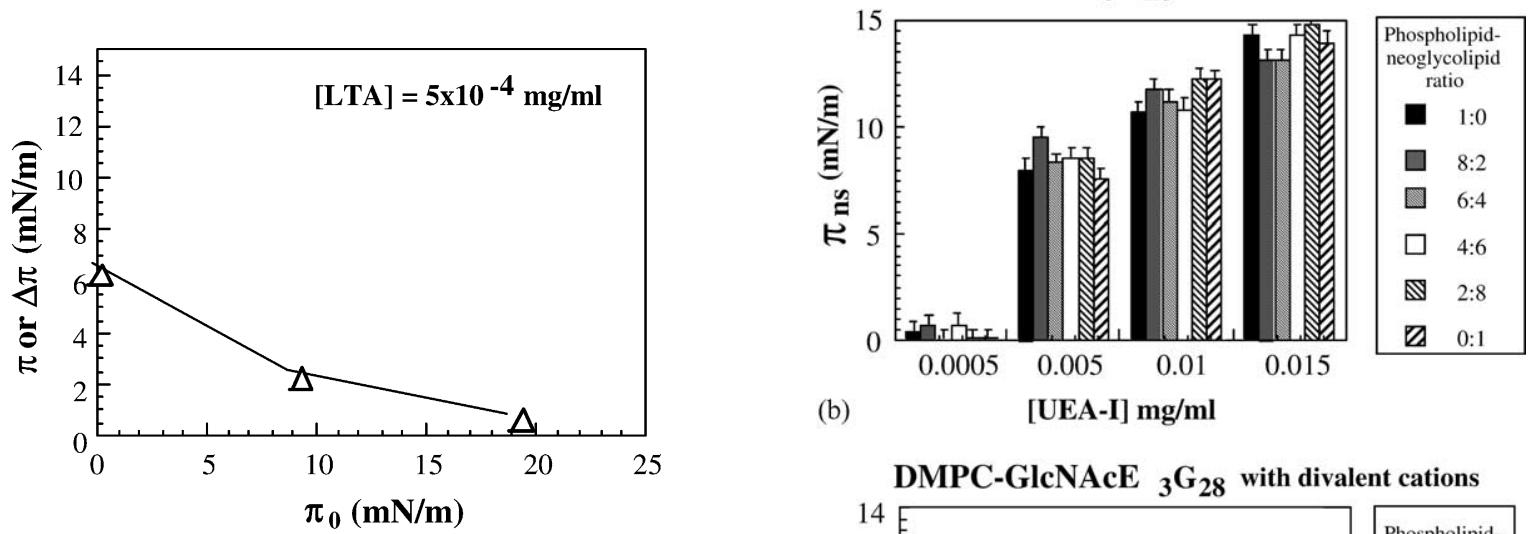

DMPC-GICNAcE ${ }_{3} \mathbf{G}_{28}$ with divalent cations

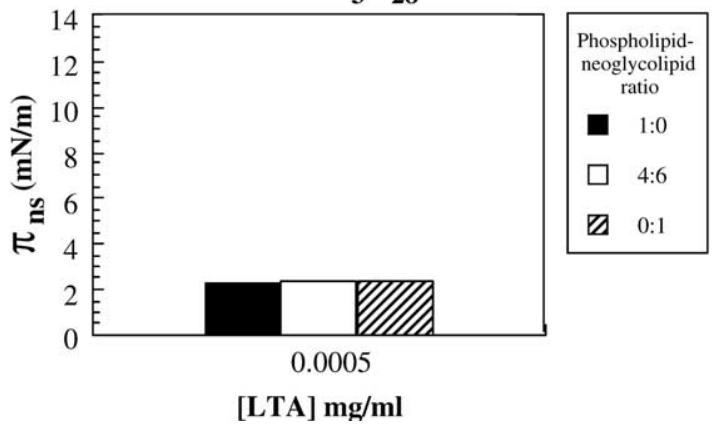

Fig. 5. Surface pressure changes in non-specific conditions $\left(\Delta \pi=\pi_{\mathrm{ns}}, \pi_{0}=9 \mathrm{mN} / \mathrm{m}\right)$. A lectin was injected beneath a DMPC/neoglycolipid (4:6) monolayer: (a) for WGA, in the absence of the essential divalent cations in the buffer solution; (b) for LTA and UEA-I, the neoglycolipid was unspecific to the lectin.

Fig. 4. Surface pressure $(\pi)$ or surface pressure changes $(\Delta \pi)$, induced by lectin adsorption at the free air/solution interface $\left(\pi_{0}=0 \mathrm{mN} / \mathrm{m}\right)$ and into spread DMPC monolayers, in the presence of divalent cations.

All specific sugar-lectin interactions were performed in the presence of divalent cations in solution. The results, expressed as $\pi_{\mathrm{s}}\left(\Delta \pi-\pi_{\mathrm{ns}}\right)$ are represented in Fig. 6. At all studied WGA concentrations,

a significant surface pressure increase was observed. It should also be noted that the neoglycolipid/DMPC ratio had a strong influence on the extent of WGA adsorption and that the highest surface pressure changes were obtained when the mixed monolayers contained about $50 \%$ of $\mathrm{GlcNAcE}_{3} \mathrm{G}_{28}$. As for the UEA-I $/ \alpha-\mathrm{FucE}_{3} \mathrm{G}_{28}$ the observed $\Delta \pi$ values were 
DMPC-GlcNAcE ${ }_{3} \mathrm{G}_{28}$ with divalent cations

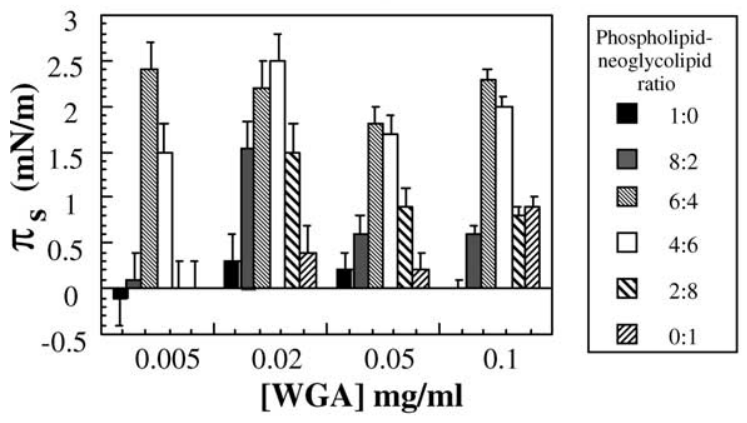

DMPC- $\alpha \mathrm{FucE}_{3} \mathrm{G}_{28}$ with divalent cations

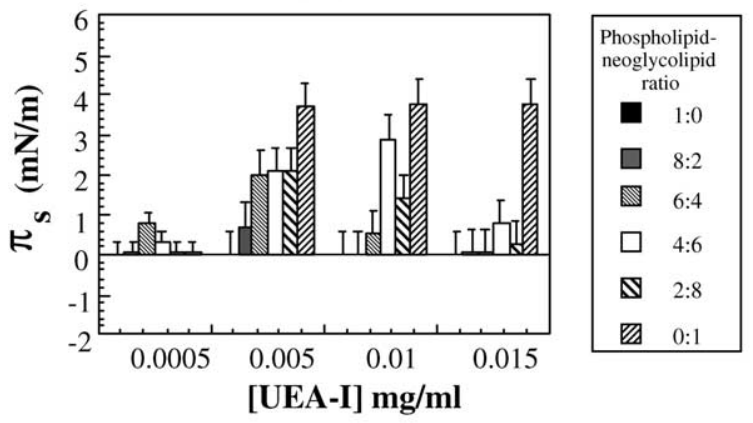

DMPC-FucE ${ }_{3} G_{28}$ with divalent cations

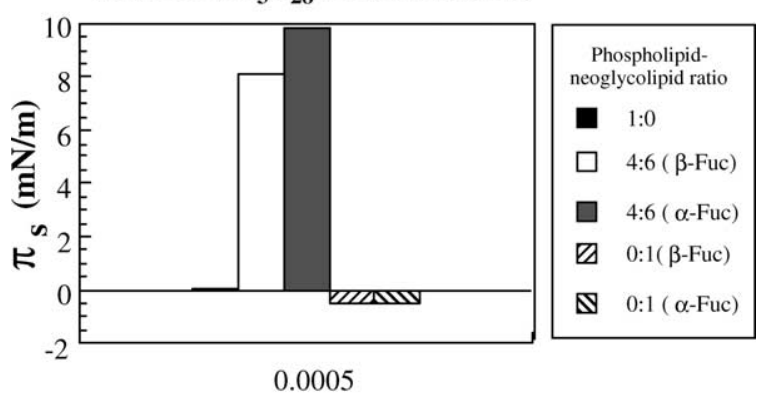

[LTA] mg/ml

Fig. 6. Surface pressure changes in specific conditions $\left(\pi_{\mathrm{s}}=\Delta \pi-\pi_{\mathrm{ns}}, \pi_{0}=9 \mathrm{mN} / \mathrm{m}\right)$. All studied lectins were injected beneath a DMPC/neoglycolipid (4:6) monolayer, in which the neoglycolipid was specific to the lectin, and in the presence of the three divalent cations in the buffer solution.

directly increased with the $\alpha$-fucose molar fraction in the mixed monolayers. The highest adsorption of UEA-I was obtained with the pure $\alpha-\mathrm{FucE}_{3} \mathrm{G}_{28}$ monolayer. Moreover, at the highest studied UEA-I solution concentration, enhanced lectin adsorption appeared only with a monolayer constituted of the pure neoglycolipid. The adsorption of both LTA and WGA into mixed monolayers was comparable and the obtained surface pressure changes were higher than their values with the pure neoglycolipids.

\subsection{Compression of monolayers}

Mixed Langmuir films of DMPC/GlcNAcE ${ }_{3} \mathrm{G}_{28}$ were compressed after lectin adsorption. These experiments made it possible to evaluate whether desorption of adsorbed lectin from the monolayer occurred or not. The recorded $\pi-A$ isotherms clearly showed that, above $28 \mathrm{mN} / \mathrm{m}$, the isotherm corresponding to $\mathrm{DMPC} / \mathrm{GlcNAcE}_{3} \mathrm{G}_{28}$ in the presence of WGA was almost identical to that of DMPC/GlcNAcE $\mathrm{E}_{3} \mathrm{G}_{28}$ spread on water (in the absence of the lectin). This would strongly suggest that the lectin was displaced from the monolayer as the result of compression (Fig. 7). As for UEA-I and LTA they both withstood their compression and remained in monolayers even at low molecular areas.

\section{Discussion}

Using the monolayer approach, we have tried to better understand how lectins adsorb on liposomes walls and what are the main factors that contribute to the agglutination of neoglycolipid-bearing vesicles.

For these reasons we have first plotted the surface pressure versus time (data not shown) according to the equation $\ln \left[\left(\pi_{\mathrm{e}}-\pi\right) /\left(\pi_{\mathrm{e}}-\pi_{0}\right)\right]=-k_{\mathrm{i}} t$, in which $\pi$ and $\pi_{\mathrm{e}}$ are the interfacial surface pressures at time $t$ and at equilibrium, respectively, and $\pi_{0}$ is the initial surface pressure. This representation takes into account the dependence of the adsorption kinetics on the rate constant $k_{\mathrm{i}}$. The obtained relationships exhibited three linear regions. Whereas the initial slope yielded a first-order rate constant of adsorption $\left(k_{1}\right)$, the second one corresponded to a first-order rate constant of protein penetration-rearrangement $\left(k_{2}\right)$. The slope corresponding to the third linear region $\left(k_{3}\right)$ was due to the rearrangement of protein molecules in the monolayer.

From the results summarized in Table 1 it may be inferred that despite its rather low surface tension effectiveness, UEA-I exhibited the highest rate constants and was characterized by the shortest overall adsorption process. This would suggest that UEA-I 

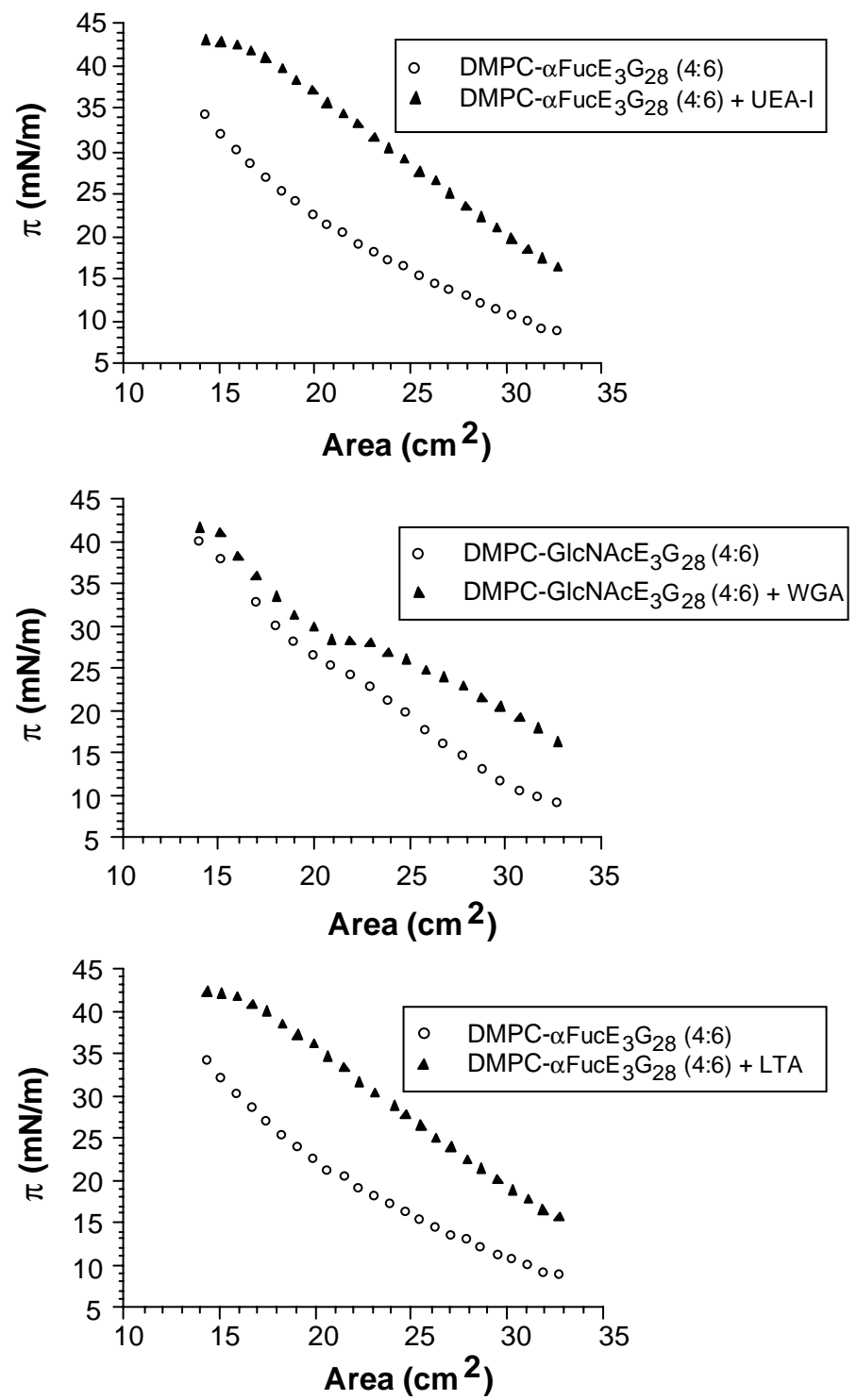

Fig. 7. Comparison of the $\pi-A$ isotherms for DMPC/neoglycolipid monolayers compressed on a Langmuir trough, in the absence and in the presence of a lectin in the subphase.

rearranged much faster than the two other lectins. Also the absence of any lag-time in adsorption kinetics supports the hypothesis of limited conformational changes for this lectin at the air/water interface. In fact, contrary to those required for WGA and LTA, relatively high surfactant or alcohol concentrations were usually necessary to denature UEA-I (Jirgensons and Ross, 1982). The LTA, which exhibits the highest surface pressure
( $\pi=15.3 \mathrm{mN} / \mathrm{m}$ at the studied concentration), is the biggest lectin in size out of the three proteins. It has a dimeric or even tetrameric structure, depending on the nature of the isolectin (Kalb, 1968). Since the rearrangement of monomeric structures at the air/water interface is generally more rapid than that of polymeric ones (Suttiprasit et al., 1992), LTA would be expected to reach its equilibrium conformation state 
Table 1

Adsorption kinetics of the three studied lectins $\left(5 \times 10^{-3} \mathrm{mg} / \mathrm{ml}\right)$ at the air/water interface, as deduced from the plots $\left[\ln \left(\pi_{\mathrm{e}}-\pi\right) / \pi_{\mathrm{e}}\right]=-k_{\mathrm{i}} t$ at $\pi_{0}=0 \mathrm{mN} / \mathrm{m}, \pi_{\mathrm{e}}$ is the surface pressure at equilibrium, $t_{\mathrm{e}}$, the time to equilibrium, $k_{1}, k_{2}, k_{3}$ are rate constants related to time periods $t_{1}, t_{2}, t_{3}$

\begin{tabular}{|c|c|c|c|c|c|c|c|c|c|}
\hline & \multicolumn{3}{|c|}{ Whole process } & \multicolumn{2}{|l|}{ Adsorption } & \multicolumn{2}{|l|}{ Penetration } & \multicolumn{2}{|c|}{ Rearrangement } \\
\hline & $\pi_{\mathrm{e}}(\mathrm{mN} / \mathrm{m})$ & $t_{\mathrm{e}}(\mathrm{h})$ & Lag-time (h) & $k_{1}\left(\min ^{-1}\right)$ & $t_{1}(\mathrm{~h})$ & $k_{2}\left(\min ^{-1}\right)$ & $t_{2}(\mathrm{~h})$ & $k_{3}\left(\min ^{-1}\right)$ & $t_{3}(\mathrm{~h})$ \\
\hline WGA & 3.5 & 21 & 0.7 & $4.82 \times 10^{-4}$ & 8.3 & $2.42 \times 10^{-3}$ & 9.0 & $1.11 \times 10^{-2}$ & 3.0 \\
\hline UEA-I & 1.8 & 15 & 0 & $3.08 \times 10^{-3}$ & 8.0 & $4.62 \times 10^{-3}$ & 4.2 & $1.21 \times 10^{-2}$ & 2.8 \\
\hline LTA & 15.3 & 40 & 1.0 & $1.34 \times 10^{-3}$ & 13.0 & $5.21 \times 10^{-4}$ & 19.0 & $8.64 \times 10^{-3}$ & 7.0 \\
\hline
\end{tabular}

after longer periods of time than UEA-I and WGA, as shown in Table 1.

It seems reasonable to consider that DMPC monolayers act as a barrier that hinders lectin adsorption. The results in Fig. 4 support this assumption for WGA and LTA. Indeed, for both these lectins, the increase in the initial surface pressure of a phospholipid monolayer resulted in a significant decrease in the surface pressure change. Conversely, for UEA-I, an important increase in the surface pressure was observed at $9 \mathrm{mN} / \mathrm{m}$, when the lectin was adsorbed onto a DMPC monolayer. Such a behavior has already been described for other proteins such as glucose oxidase, for example, and has been attributed to strong hydrophobic interactions between the protein and phospholipid chains (Rosilio et al., 1997; Zhang et al., 2000; Li et al., 2003).

From the data obtained in the non-specific conditions (i.e. the interaction of lectins with a monolayer in the absence of a specific sugar or of divalent cations, Fig. 5), we have deduced the contribution of the non-specific interaction $\left(\pi_{\mathrm{ns}}\right)$ to the overall surface pressure changes $\left(\Delta \pi=\pi_{\mathrm{ns}}=\pi_{\mathrm{T}}-\pi_{0}\right)$. It was then possible to evaluate the contribution of the specific interaction $\left(\pi_{\mathrm{s}}=\Delta \pi-\pi_{\mathrm{ns}}\right)$ to the surface pressure responses obtained after injection of these lectins beneath the monolayers containing the specific sugars (Fig. 6). The results obtained from these experiments evidenced different mechanisms involved in interaction of the three studied lectins depending on the phospholipid/neoglycolipid ratio. Thus, for WGA, a rather low adsorption at higher DMPC/neoglycolipid ratios than the (1:1) ratio may be attributed (i) to an unfavorable change in conformation of WGA after its binding to the sugar moieties that would hinder its penetration into the monolayer, (ii) to the stripping of some of neoglycolipid molecules from the interface, due to their interaction with several binding sites of WGA. Conversely, for UEA and LTA, the $\pi_{\mathrm{s}}$ values increased with the neoglycolipid content in spread monolayers.

Three aspects have been considered: (i) the possible conformation changes in the lectins following their interaction with sugar moieties, (ii) the contribution from hydrophobic interactions between the lectin and the spread lipids, and (iii) the effect of an increase in the neoglycolipid ratio in the mixed monolayer.

The kinetics of adsorption of the three lectins in the presence and in the absence of the specific neoglycolipids in the spread monolayer have been analyzed from the $\pi$-time relationships (data not shown). The results of the calculations are presented in Table 2. The UEA-I-DMPC/ $\alpha-\mathrm{FucE}_{3} \mathrm{G}_{28}$ (4:6) system yielded a unique $k$ value equal to $4.77 \times 10^{-3} \mathrm{~min}^{-1}$. This constant represents a combined value of adsorption and penetration processes at the interface. The time periods corresponding to both the adsorption-penetration and rearrangement steps were shortened in the presence of the saccharide moiety. From these calculations it was apparent that the specific interaction between UEA-I and its ligand led to almost simultaneous adsorption and penetration processes. From the results in Figs. 4-6 and from the calculations in Table 2, it was also obvious that in our experimental conditions, in which all sugar moieties were located below the interface, the interaction between UEA-I and the spread molecules was driven by both its high non-specific hydrophobic interaction with the lipid chains of the phospholipid or neoglycolipid molecules, and its specific interaction with the fucose moieties. All seems to indicate that the observed interaction was due to the existence of the cooperative effect of adsorption and molecular recognition processes. Moreover, once the lectin has penetrated into the monolayer, strong hydrophobic interactions with the spread lipids would 
Table 2

Rate constants for the three studied lectins adsorbed into (1) pure DMPC and (2) DMPC/specific neoglycolipid (4:6) mixed monolayers

\begin{tabular}{|c|c|c|c|c|c|c|}
\hline \multirow[t]{2}{*}{ Rate constant } & \multicolumn{2}{|l|}{ UEA-I } & \multicolumn{2}{|l|}{ LTA } & \multicolumn{2}{|l|}{ WGA } \\
\hline & 1 & 2 & 1 & 2 & 1 & 2 \\
\hline$k_{1}\left(\min ^{-1}\right)$ & $1.66 \times 10^{-3} / 12 \mathrm{~h}$ & $4.77 \times 10^{-3} / 13 \mathrm{~h}$ & $3.66 \times 10^{-3} / 4 \mathrm{~h}$ & $1.05 \times 10^{-3} / 18 \mathrm{~h}$ & $2.23 \times 10^{-3} / 5 \mathrm{~h}$ & $8.78 \times 10^{-4} / 7 \mathrm{~h}$ \\
\hline$k_{2}\left(\min ^{-1}\right)$ & $3.64 \times 10^{-3} / 6 \mathrm{~h}$ & & $5.28 \times 10^{-4} / 30 \mathrm{~h}$ & $1.73 \times 10^{-3} / 17 \mathrm{~h}$ & $4.32 \times 10^{-3} / 3.5 \mathrm{~h}$ & $3.57 \times 10^{-3} / 12 \mathrm{~h}$ \\
\hline$k_{3}\left(\min ^{-1}\right)$ & $1.34 \times 10^{-2} / 3 \mathrm{~h}$ & $1.16 \times 10^{-3} / 2 \mathrm{~h}$ & $2.09 \times 10^{-3} / 12 \mathrm{~h}$ & $5.80 \times 10^{-3} / 5 \mathrm{~h}$ & $7.85 \times 10^{-3} / 5 \mathrm{~h}$ & $1.80 \times 10^{-2} / 2 \mathrm{~h}$ \\
\hline
\end{tabular}

stabilize the protein within a monolayer as shown by the profiles of the compression experiments in Fig. 7.

For WGA, the rate constants $k_{1}$ and $k_{2}$ associated with the adsorption-penetration of the lectin into a DMPC monolayer, decreased in the presence of $\mathrm{GlcNAcE}_{3} \mathrm{G}_{28}$ (Table 2). Obviously, WGA adsorption was delayed by the interaction between the lectin and its specific sugar. Other published data (Bonnin et al., 1999) showed that WGA binding to $\mathrm{GlNAcE}_{3} \mathrm{G}_{28}$ induced conformational changes in the protein backbone. It is reasonable, therefore, to think that after its interaction with the neoglycolipid, WGA becomes structurally altered. Its low $\Delta \pi$ would be in favor of this argument. Another argument in favor of the conformational change is that WGA was squeezed out of the monolayer during compression (Fig. 7). Indeed, this result demonstrates that the lectin did not strongly interact with the hydrophobic tails of the spread lipids and could not remain at the interface when the monolayer was confined to smaller molecular areas.

For LTA, as for WGA, the $k_{1}$ values decreased in the presence of the neoglycolipid while the adsorption time dramatically increased. However, contrarily to WGA, the overall time period of adsorption and penetration processes $(35 \mathrm{~h})$ was identical to that in the absence of the neoglycolipid ( $34 \mathrm{~h}$ ) (Table 2). This would mean that whereas the adsorption/penetration of LTA was delayed by its interaction with the sugar determinant, the rearrangement of the lectin-saccharide complex in the monolayer occurred faster than that of the lectin alone. As for WGA, the binding of LTA molecules to their sugar determinants triggered most probably some conformational changes that affected its adsorption at the interface. However, the compression experiments (Fig. 7) showed that contrarily to WGA, LTA remained in the monolayer during compression.

Another point should be stressed here. In both the surface and solution experiments, the interaction be- tween lectins and neoglycolipids was generally favored by an increase in the sugar molar fraction. However, if the adsorption of UEA-I to the pure neoglycolipid led to the highest change in surface pressure, for WGA and LTA their binding to the monolayers containing high sugar molar fractions was limited and for pure neoglycolipid films even drastically reduced (Fig. 6). Such differences between UEA-I and the two other studied lectins may be attributed to the differences in their respective accessibilities to the sugar moieties and to the hydrophobic lipid chains. Table 3 summarizes the values of calculated surface areas corresponding to fucose and $\mathrm{N}$-acetylglucosamine moieties (data from Faivre et al., 2001). The comparison of the data from Fig. 6 and Table 3 clearly indicates that at high neoglycolipid molar fractions, the larger the available space in the vicinity of the specific sugar, the higher WGA or LTA binding. The accessibility of these lectins to the sugar moiety seems, therefore, to be the only determining factor of their interaction with the monolayer. For UEA-I, one can hypothesize that its interaction with the mixed monolayers is controlled mainly by hydrophobic interactions at the level of the lipid chains. Indeed, the reduction of the surface area per sugar moiety from 200 to $88 \AA^{2}$ did not affect lectin adsorption (Table 3 ).

In summary, our results show that for UEA-I its binding to both the monolayers and the liposomes bearing its specific sugar determinant is boosted by

Table 3

Effect of the composition of a monolayer on the size of the available area around the sugar headgroups

\begin{tabular}{lll}
\hline $\begin{array}{l}\text { Composition of the } \\
\text { monolayer }\end{array}$ & \multicolumn{2}{c}{ Available surface area $\left(\AA^{2}\right)$} \\
\cline { 2 - 3 } & $\alpha-\mathrm{FucE}_{3} \mathrm{G}_{28}$ & $\mathrm{GlcNAcE}_{3} \mathrm{G}_{28}$ \\
\hline $\begin{array}{l}\text { DMPC/neoglycolipid (4:6) } \\
\text { Pure neoglycolipid }\end{array}$ & 204 & 212 \\
\hline
\end{tabular}


a combined effect of molecular recognition that takes place and strong hydrophobic non-specific interaction of lectin molecules that at least partially penetrate into the lipid wall. Conversely, for WGA and LTA, the size of the available space around each sugar group seems to play a key role in neoglycolipid recognition by those lectins. For these two lectins, the specific interactions most probably entail a change in the lectin conformations that alters their surface properties.

\section{Acknowledgements}

This work was supported by the French Embassy in Portugal-ICCTI agreement (Project No. 018K4).

\section{References}

Ahlers, M., Müller, W., Reichert, A., Ringsdorf, H., Venzmer, J., 1990. Specific interactions of proteins with functional lipid monolayers - ways of simulating biomembranes processes. Angew Chem. Int. Ed. Engl. 29, 1269-1285.

Akiyoshi, K., Takanabe, H., Sato, T., Kondo, H., Sunamoto, J., 1990. Cell specificity of polysaccharide derivatives on liposomal surface. Chem. Lett. 473-476.

Allen, H.J., Johnson, E.A.Z., Matta, K.L., 1977. A comparison of the binding specificities of lectins from Ulex europaeus and Lotus tetragonolobus. Immunol. Comm. 6, 585-602.

Ashwell, G., Morell, A.G., 1974. The role of surface carbohydrates in the hepatic recognition and transport of circulating glycoproteins. In: Meister, A. (Ed.), Advance in Enzymology. John Wiley, New York, pp. 99-128.

Baszkin, A., Norde, W., 1999. Physical Chemistry of Biological Interfaces. Marcel Dekker, New York.

Berthelot, L., Rosilio, V., Costa, M.L., Chierici, S., Albrecht, G., Boullanger, P., Baszkin, A., 1998. Behavior of amphiphilic neoglycolipids at the air/solution interface. Interaction with a specific lectin. Colloids Surf. B 11, 239-248.

Blanzat, M., Perez, E., Rico-Lattes, I., Prome, D., Prome, J.C., Lattes, A., 1999. New cationic glycolipids. 1. Synthesis characterization and biological activity of double-chain and gemini cationic analogs of galactosylceramide (gal $\beta_{1}$ cer). Langmuir 15, 6163-6169.

Boldt, D.H., Speckart, S.F., Richards, R.L., Alving, C.R., 1977. Interactions of lectins with glycolipids in liposomes. Biochem. Biophys. Res. Commun. 74, 208-214.

Bonnin, S., Besson, F., Gelhausen, M., Chierici, S., Roux, B., 1999. A FTIR spectroscopy evidence of the interactions between wheat germ agglutinin and $\mathrm{N}$-acetylglucosamine residues. FEBS Lett. 456, 361-364.

Boullanger, P., Sancho-Camborieux, M.R., Bouchu, M.N., Marron-Brignone, L., Morelis, P.R., Coulet, R.M., 1997. Synthesis and interfacial behavior of three homologous glyceroneoglycolipids with various chain lengths. Chem. Phys. Lipids 90, 63-74.

Chen, H., Torchilin, V., Langer, R., 1996. Lectin-bearing polymerized liposomes as potential oral vaccine carriers. Pharm. Res. 13, 1378-1383.

Chierici, S., Boullanger, P., Marron-Brignone, L., Morelis, R.M., Coulet, P.R., 1997. Synthesis and interfacial behavior of a gemini neoglycolipid. Chem. Phys. Lipids 87, 91-101.

Emmanouil, V., El Ghoul, M., André-Barrès, C., Guidetti, B., Rico-Lattes, I., Lattes, A., 1998. Synthesis of new long-chain fluoroalkyl glycolipids: relation of amphiphilic properties to morphology of supramolecular assemblies. Langmuir 14, 5389-5395.

Esumi, K., Hosoya, T., Suzuki, A., Torigoe, K., 2000. Spontaneous formation of gold nanoparticles in aqueous solution of sugar-persubstituted poly(amidoamine) dendrimers. Langmuir 16, 2978-2980.

Faivre, V., Manivet, P., Callaway, J.C., Airaksinen, M.M., Morimoto, H., Baszkin, A., Launay, J.M., Rosilio, V., 2000. Ligand interaction with purified serotonin transporter in solution and at the air/water interface. FEBS Lett. 471, 56-60.

Faivre, V., Rosilio, V., Boullanger, P., Martins-Almeida, L., Baszkin, A., 2001. Fucosyled neoglycolipids: synthesis and interaction with a phospholipid. Chem. Phys. Lipids 109, 91101.

Feizi, T., 1985. Demonstration by monoclonal antibodies that carbohydrate structures of glycoproteins and glycolipids are onco-developmental antigens. Nature 314, 53-57.

Hare, B.J., Rise, F., Aubin, Y., Prestegard, J.H., $1994 .{ }^{13} \mathrm{C}$ NMR studies of wheat germ agglutinin interactions with $\mathrm{N}$-acetylglucosamine at a magnetically oriented bilayer surface. Biochemistry 33, 10137-10148.

Irache, J.M., Durrer, C., Duchêne, D., Ponchel, G., 1994. In vitro study of lectin-latex conjugates for specific bioadhesion. J. Control Release 31, 181-188.

Jirgensons, B., Ross, D., 1982. Circular dichroism studies on conformational transitions of phytohemagglutinins effected by some alcohols. Int. J. Pept. Protein Res. 20, 110-114.

Kalb, A.J., 1968. The separation of three L-fucose-binding proteins of Lotus tetragonolobus. Biochim. Biophys. Acta 168, 532536.

Kishore, U., Eggleton, P., Reid, K.B.M., 1997. Modular organization of carbohydrate recognition domains in animal lectins. Matrix Biol. 15, 583-592.

Kitano, H., Kuwayama, M., Kanayama, N., Ohno, K., 1998. Interfacial recognition of sugars by novel boronic acid-carrying amphiphiles prepared with a lipophilic radical initiator. Langmuir 14, 165-170.

Kurihara, K., Ohto, K., Honda, Y., Kunitake, T., 1991. Efficient, complementary binding of nucleic acid bases to diaminotriazine-functionalized monolayers. J. Am. Chem. Soc. 113, 5077-5078.

Li, J., Rosilio, V., Boissonnade, M.M., Baszkin, A., 2003. Adsorption of glucose oxidase into lipid monolayers: effect of lipid headgroups charge. Colloids Surf. B 29, 13-20.

Liener, I.E., Sharon, N., Goldstein, I.J., 1986. The Lectins: Properties, Functions and Applications in Biology and Medicine. Academic Press, Orlando. 
Monsigny, M., Kieda, C., Roche, A.C., 1979. Membrane lectins. Biol. Cellulaire 36, 289-300.

Murahashi, N., Ishihara, H., Sakagami, M., Sasaki, A., 1997. Synthesis and application of neoglycolipids for liposomes modification. Biol. Pharm. Bull. 20, 704-707.

Nagata, Y., Burger, M.M., 1974. Wheat germ agglutinin. Molecular characteristics and specificity for sugar binding. J. Biol. Chem. 249, 3116-3122.

Ponchel, G., Irache, J.M., 1998. Specific and non-specific bioadhesive particulate systems for oral delivery to the gastrointestinal tract. Adv. Drug Delivery Rev. 34, 191-219.

Roseman, S., 1970. The synthesis of complex carbohydrates by multiglycosyl transferase system and their potential function in intracellular adhesion. Chem. Phys. Lipids 5, 270-297.

Rosilio, V., Boissonnade, M.M., Zhang, J., Jiang, L., Baszkin, A., 1997. Penetration of glucose oxydase into organized phospholipid monolayers spread at the solution/air interface. Langmuir 13, 4669-4675.
Sharon, N., Lis, H., 1993. Carbohydrates in cell recognition. Sci. Am. 268, 82-89.

Sancho, M.R., Boullanger, P., Létoublon, R., 1993. Incorporation of D-glucosamine alkyl glucopyranosides into the bilayer of small unilamellar vesicles of egg phosphatidylcholine. Colloids Surf. B 1, 373-381.

Shimada, K., Kamps, J.A.A.M., Regts, J., Ikeda, K., Shiozawa, T., Hirota, S., Scherphof, G.L., 1997. Biodistribution of liposomes containing synthetic galactose-terminated diacylglyceryl-poly (ethyleneglycol)s. Biochim. Biophys. Acta 1326, 329-341.

Suttiprasit, P., Krisdhashima, V., McGuire, J., 1992. The surface activity of $\alpha$-lactalbumin, $\beta$-lactoglobulin, and bovine serum albumin. J. Colloid Interface Sci. 154, 316-325.

Zhang, J., Rosilio, V., Goldmann, M., Boissonnade, M.M., Baszkin, A., 2000. Adsorption of glucose oxidase into lipid monolayers. Effect of lipid chain lengths on the stability and structure of mixed enzyme/phospholipid films. Langmuir 16, 12261232. 